\title{
Delirium in COVID-19 patients: a multicentric observational study in Italy
}

\author{
Giovanni Martinotti ${ }^{1,2} \cdot$ Laura Bonanni $^{1} \cdot$ Stefano Barlati ${ }^{3} \cdot$ Andrea Miuli $^{4}\left({ }^{\circ} \cdot\right.$ Gianna Sepede $^{1} \cdot$ Davide Prestia $^{5}$. \\ Alice Trabucco ${ }^{5}$. Claudia Palumbo ${ }^{6}$. Alessandra Massaro $^{6} \cdot$ Martina Olcese $^{7} \cdot$ Damiano D'Ardes $^{8}$. \\ Francesco Cipollone ${ }^{8} \cdot$ Mario Amore $^{5} \cdot$ Emi Bondi $^{6} \cdot$ Mirella Russo $^{1}$. Claudia Carrarini ${ }^{1} \cdot$ Marco Onofrj $^{1}$. \\ Stefano Luca Sensi ${ }^{1} \cdot$ Antonio Vita $^{3} \cdot$ Massimo di Giannantonio ${ }^{1}$
}

Received: 22 March 2021 / Accepted: 3 July 2021 / Published online: 28 July 2021

(C) Fondazione Società Italiana di Neurologia 2021

\begin{abstract}
Introduction Although recent data show that SARS-CoV-2 infection seems to affect the central nervous system (CNS), little is known about the neuropsychiatric effects resulting from this condition. In addition to the well-known neurotrophism of coronaviruses, recent evidence shows also that the "cytokine storm" induced by the infection is at the basis of the neuroinflammation of the CNS. Furthermore, prolonged hospitalization, polypharmacotherapy, and isolation could be at the basis of the onset of delirium in hospitalized COVID patients. This multicentric observational study explores the incidence of the onset of delirium in an Italian cohort of SARS-CoV-2 positive inpatients.

Methods Data were collected in the COVIDhospitals of Brescia, Bergamo, Chieti, and Genova. Different socio-demographic, medical, neurological, and pharmacological parameters were collected. As a rapid screening for delirium, the 4AT scale was used. Eighty COVID-19 inpatients (mean age $74.7 \pm 14.5$ years) met the inclusion criteria (confirmed positivity to the SARS-CoV-2 virus; the presence of delirium and/or psychomotor agitation and/or new onset of other neuropsychiatric symptoms during hospitalization).

Results Themajority of these patients (68.8\%) had "hyperactive delirium" subtype. Polypharmacotherapy, current treatment with corticosteroids, and higher age were associated with delirium severity.

Conclusion These data provide an insight into the onset of delirium among COVID-19 patients underlining the need for monitoring, especially in elderly patients, the neuropsychiatric symptoms, and the therapy in order to have shorter hospitalization times and better outcomes.
\end{abstract}

Keywords SARS-CoV-2 infection $\cdot$ COVID-19 $\cdot$ Delirium $\cdot$ Neuroinflammation

Antonio Vita and Massimo di Giannantonio are equally contributed as last author.

Andrea Miuli

andreamiuli@live.it

1 Department of Neuroscience, Imaging and Clinical Sciences, "G. D’Annunzio" University of Chieti, Chieti, Italy

2 Department of Pharmacy, Pharmacology, Clinical Science, University of Hertfordshire, Hatfield, Herts, UK

3 Department of Clinical and Experimental Sciences, University of Brescia, Brescia, Italy

4 Department of Mental Health, ASL Lanciano-Vasto-Chieti, Viale Amendola 47, Chieti, Italy
5 Department of Neuroscience, Ophthalmology, Genetics and Infant-Maternal Science, Section of Psychiatry, University of Genoa, Genoa, Italy

6 Department of Psychiatry, Hospital Papa Giovanni XXIII, Bergamo, Italy

7 Psychologist, Independent Researcher, Genoa, Italy

8 Department of Medicine and Aging Sciences, University G. D'Annunzio of Chieti-Pescara, Chieti, Italy 


\section{Introduction}

\section{Neurologic tropism and clinical pictures associated with SARS-CoV-2 infection}

The severe acute respiratory syndrome associated with coronavirus 2 (SARS-CoV-2) infection, responsible for COronaVIrus Disease 19 (COVID-19), also affects blood coagulation and the central nervous system (CNS). While COVID-19-related neurological conditions have gathered great attention, little is known about the capacity of SARS$\mathrm{CoV}-2$ to exacerbate and/or promote neuropsychiatric symptoms.

Several mechanisms have been proposed to explain the neurotropism showed by this virus. One hypothesis indicates that the virus can spread from the olfactory epithelium to the olfactory bulb (through the so-called transcribial route), then retrogradely propagate to the brain $[1,2]$. The CNS environment is highly suitable for SARS$\mathrm{CoV}-2$ invasion since neurons and glia cells express Angiotensin-Converting Enzyme 2 receptors (ACE2-R) which promote SARS-CoV-2 adhesion by the spike protein $\mathrm{S} 1$ [3]. After adhesion, viral replication cycles begin (Baig 2020), leading to neuroinflammation and neuronal damage [3]. This theory is supported by the frequent, often isolated presence of anosmia and dysgeusia as the first symptoms of the infection, independently from other viral infection symptoms [4]. Further evidence supports the notion that coronaviruses can use active intraneuronal transport to spread from the peripheral nervous system to the CNS [5] and back (Fenrich et al. 2020). Another proposed mechanism of access to the CNS is via hematogenous dissemination through the infection of some myeloid cell lines, mostly macrophages [6]. This way could be promoted by a disruption of the blood-brain-barrier (BBB), due to endothelial and pericyte viral invasion [2], a pathway relevant in immunocompromised individuals [7]. The neuronal dysregulation of the water/ionic balance and direct inflammation [2] can furtherly contribute to neurological impairment in SARS-CoV-2 infection. Systemic and CNS inflammation have been related to the onset of several COVID-19 complications, including impaired consciousness $[8,9]$. This neuroinflammation is explained by the "cytokine storm," a complex reaction of the immune system involving several pro-inflammatory cytokines, the renin-angiotensin aldosterone system and the activation of the complement cascade [10]. This pro-inflammatory pathway leads, during SARS-CoV-2 infection, to endothelial alteration, microglia dysfunction, and, also, evidences of demyelination [2].

Supporting the CSN tropism of the virus, several studies have indicated the SARS-CoV-2 presence in the brain of patients affected by epilepsy [11], cerebral edema [12], encephalitis [13], and multiple sclerosis [14]. Recent hypothesis suggest that the frontal lobes in particular could be a specific target of SARS-CoV-2 infection [15].

However, the relationship between the SARS-CoV-2 brain infections and neurologic diseases still needs to be fully assessed and an inflammatory mechanism is considered more plausibly associated to the appearance of CNS damage [16, 17]. In this regard, a recent review [18] summarizes the neuroinflammatory role of Sars-CoV-2 by analyzing the infection from the point of view of neuroimaging, electrophysiological and laboratory, underlining the ability of the virus to generate encephalitis especially in the elderly.

Recent studies have explored delirium incidence [19] in patients suffering from severe acute respiratory distress, and treated in intensive care units (ICU) and isolation settings [20]. Delirium is a state of acute confusion characterized by mental status changes associated with altered consciousness levels, impaired attention and concentration, and disorganized thinking [21]. Hallucinations, illusions, or delusions may also occur. For some patients, delirium is reversible within days. However, the condition can also be the herald of permanent cognitive and behavioral impairment, especially in patients already prone to neurodegenerative processes. Full-blown delirium associated with intense agitation in a combative patient is often described as "hyperactive delirium." The only increase in motor/emotional and behavioral activity is called psychomotor agitation (PA), and it could be considered only as an aspect of the complex manifestation of an hyperactive delirium [22]. In contrast, sometimes patients exhibit a very quiet and subdue behavior, a condition called "hypoactive delirium" characterized by drowsiness, a reduction in motor activity/inactivity. A "mixed" presentation can also occur. Early observations indicated that $20-30 \%$ of COVID-19 patients present with or develop delirium during their hospitalization [23] with rates of 55-70\% in cases of severe illness [24].

It should be noted that some first-line medications for COVID-19, like antiretrovirals, can cause or contribute to delirium [25]. Considering the high costs [26] and the high specialization required to deal with acute neuropsychiatric cases, this study explores the incidence of delirium and other neuropsychiatric symptoms (NPS) in an Italian cohort of SARS-CoV-2 positive inpatients. The study also investigates the therapeutic efficacy, in delirium treatment, for different pharmacological classes.

\section{Materials and methods}

\section{Patients' enrolment}

This survey was conducted in "SS. Annunziata" hospital of Chieti, "Papa Giovanni XXIII" hospital of Bergamo, 
"ASST Spedali Civili" of Brescia, and "S.Martino" hospital of Genoa, to evaluate the socio-demographic and clinical characteristics of SARS-CoV-2 positive patients with delirium, PA, or other NPS.

Inclusion criteria were (1) confirmed positivity to the SARS-CoV-2 virus (with PCR technique), (2) the presence of delirium (hyperactive, hypoactive, mixed), and/or (3) PA and/or new onset NPS (delusions, hallucinations), described on at least one occasion during hospitalization.

Exclusion criteria were (1) a previous diagnosis of major psychiatric disorders (schizophrenic spectrum disorders) and (2) a prior diagnosis of primary or secondary dementia.

\section{Clinical assessment}

The following parameters were evaluated: age; sex; neurological conditions; concomitant therapy; concomitant COVID-19 symptomatology during the occurrence of NPS; the presence of invasive or non-invasive mechanical ventilation during NPS; narrative description and clinical classification of the acute NPS syndromes and symptoms (hyperactive/hypoactive/mixed delirium, psychomotor agitation, aggression, delusions, hallucinations, anti-conservative ideation (an articulate expression of a suicidal intent), other; pharmacological treatment of the acute NPS conditions. As a rapid screening for delirium, the " 4 "A's Test" (4AT) was used [27]. In few minutes, the questionnaire evaluates the general cognitive status and does not require the patient to answer questions directly. No specific training is needed to administer the questionnaire.

Neuropsychological assessment at the admission was performed by medical staff in all the patients who were not critically ill or intubated.

Afterwards, the patients were divided into three groups according to the cognitive level, i.e., cognitively normal (CN), mild cognitive impairment (MCI), and patients with dementia (PwD). Outcomes of the illness (survived/ deceased) were provided for the thirty-eight patients recruited by the Center of Brescia.

All observations were made according to the principles of good clinical practice and research according to the Helsinki Convention [28]. All data were collected and anonymized.

This protocol was approved by the Ethics Committee of the Ospedali Civili of Brescia as a promoter center; the other centers are included as follower centers.

\section{Statistical analysis}

The statistical analysis was performed using Statistica 8.0 (Statsoft Inc. USA, 2007). Quantitative parameters were presented as mean \pm standard deviation (SD) and qualitative parameters as number and percentage per class. Kolmogorov-Smirnov (K-S test) was used to check for normality of distributions. Chi-square test was used for categorical variables, and Fisher exact two-tailed test was applied in case of frequencies $<5$. When comparing the means of two independent groups, we used a two-sample t-test. Analysis of variance (ANOVA) and Duncan post hoc test were used for continuous variables in three or more independent groups. Analysis of covariance (ANCOVA) was ran in order to control for the effects of selected continuous variables, which co-vary with the dependent variable. The statistical threshold was set at $p<0.05$. To analyze the relations among delirium severity, measured by 4AT, and demographic and clinical variables, a Generalized Regression model (GRM) was employed: 4AT was set as the dependent variable, whereas age, gender, mean number of comorbid conditions, and mean number of pharmacological treatments were set as predictors.

\section{Results}

\section{Descriptive statistics}

Eighty COVID-19 inpatients (mean age $74.7 \pm 14.5$ years), showing the clinical features of delirium, were recruited by four Italian Clinical COVID-19 Centers (Bergamo, Brescia, Chieti, Genova). Table 1 shows all the demographic, clinical, and therapeutical features of the cohort. Forty-five patients (56.2\%) underwent neuropsychological screening for cognitive deficits at admission. This condition was not evaluated in other patients due to their clinical status (Table 1).

Treatment with corticosteroids and antipsychotics was associated with a significantly higher score at 4AT than the other subjects (Table 2). The GRM model accounted for about $17 \%$ of the variance $(R$ square $=0.17 ; p<0.01)$ and a significant effect was observed for age (beta $=0.34$; $t=2.8 ; p=0.02)$ and number of pharmacological treatments (beta $=0.30 ; t=2.6 ; p=0.01$ ), whereas the effect of gender (beta $=0.15 ; \mathrm{t}=1.3 ; p=0.19)$ and number of comorbid conditions (beta $=0.18 ; t=1.6 ; p=0.12$ ) was not significant. Hence, higher age and number of pharmacological drugs were significantly associated to higher 4AT scores.

\section{Exploratory analyses in data subsets}

1) Cognitive assessment: a three-group ANOVA was performed in those subjects who had undergone cognitive screening at the admission, with 4AT score set as the dependent variable. Surprisingly, the 4AT score was higher in MCI subjects than PwD (Table 3, Fig. 1).

2) Long-term outcome (survived, deceased): out of a total of thirty-eight subjects evaluated, twenty-eight died (73.7\%). When compared for delirium severity, the deceased patients' 4AT scores were higher than the sur- 
Table 1 Demographic and clinical variables

\begin{tabular}{|c|c|c|}
\hline & Mean & SD \\
\hline \multirow[t]{2}{*}{ Age } & 74.7 & 14.5 \\
\hline & $N$ & $\%$ \\
\hline \multicolumn{3}{|l|}{ Gender } \\
\hline Female & 30 & 37.5 \\
\hline Male & 48 & 60.0 \\
\hline Missing data & 2 & 2.5 \\
\hline \multicolumn{3}{|l|}{ Hospital unit } \\
\hline Chieti & 14 & 17.5 \\
\hline Genova & 15 & 18.8 \\
\hline Bergamo & 13 & 16.3 \\
\hline Brescia & 38 & 47.5 \\
\hline \multicolumn{3}{|l|}{ Delirium subtype } \\
\hline Hyperactive & 55 & 68.8 \\
\hline Hypoactive & 18 & 22.5 \\
\hline Mixed & 7 & 8.7 \\
\hline \multicolumn{3}{|l|}{ Cognitive level at admission } \\
\hline Normal & 25 & 31.3 \\
\hline Mild cognitive impairment & 12 & 15.0 \\
\hline Dementia & 8 & 10.0 \\
\hline Missing data & 35 & 43.8 \\
\hline \multicolumn{3}{|l|}{ Reason for admission } \\
\hline Infectious & 68 & 85.0 \\
\hline Infectious + pneumological & 7 & 8.8 \\
\hline Infectious + psychiatric & 1 & 1.3 \\
\hline Infectious + pneumological + neurological & 1 & 1.3 \\
\hline Infectious + neurological & 1 & 1.3 \\
\hline Gastrointestinal & 2 & 2.5 \\
\hline \multirow[t]{2}{*}{ Cardiological + pneumological } & 2 & 2.5 \\
\hline & Mean & SD \\
\hline \multirow[t]{2}{*}{ Comorbid conditions } & 3.1 & 1.5 \\
\hline & $N$ & $\%$ \\
\hline At least one comorbid condition & 78 & 97.5 \\
\hline Cardiological & 52 & 65.0 \\
\hline Pneumological & 19 & 23.8 \\
\hline Neurological & 11 & 13.8 \\
\hline Psychiatric & 9 & 11.3 \\
\hline Oncological & 8 & 10.0 \\
\hline Autoimmune & 4 & 5.0 \\
\hline \multirow[t]{2}{*}{ Other } & 15 & 18.8 \\
\hline & Mean & SD \\
\hline \multirow[t]{2}{*}{ Pharmacological treatments } & 2.8 & 1.2 \\
\hline & $N$ & $\%$ \\
\hline Heparin & 44 & 55.0 \\
\hline Hydroxychloroquine & 45 & 56.3 \\
\hline Antivirals & 30 & 37.5 \\
\hline Antibiotics & 58 & 72.5 \\
\hline Corticosteroids & 39 & 48.8 \\
\hline Antipsychotics & 19 & 23.8 \\
\hline Benzodiazepines & 26 & 32.5 \\
\hline Antidepressants & 7 & 8.8 \\
\hline
\end{tabular}

vivors' (mean 10.7 vs. 8.4 ; ANOVA $\mathrm{F}=7.7 ; p<0.01$ ). However, the dead patients were also significantly older than those who survived (mean age 79.8 vs. 71.5 years; $p<0.01)$. Using age as a covariate in an ANCOVA design, the difference in 4AT scores was no more statistically significant $(p=0.085$, see Table 4$)$. Heparin treatment did not influence the mortality (no. of deceased subjects: $19 / 25$ vs. $9 / 13$; chi-square $=0.2$; Fisher exact two-tailed $p=0.71$ ).

3) Heparin and corticosteroid treatment: forty-four subjects (55\%) were treated with heparin and thirty-nine (48.8\%) with corticosteroids. While the heparin treatment did not show any significance with 4AT scores $(p=0.23$, see Table 4), the patient treated with corticosteroid therapy showed a significative relation with the severity of 4AT $(p=0.01$, see Table 4$)$.

\section{Discussion}

These data provide an insight into the onset of delirium among COVID-19 patients. Delirium severity was associated with older age and number of drugs at the evaluation point, thus revealing relevant factors that should be considered in COVID-19 units. Hyperactive delirium was the most common presentation, in line with trends reported in the elderly and the ICU [29].

Patients suffering from MCI showed greater severity of delirium-related symptoms, as assessed by the 4AT. Since a strict correlation between cognitive impairment and delirium onset in hospitalized subjects is well-acknowledged [30], this result was expected. Conversely, the lower 4AT scores observed $\mathrm{PwD}$, compared to $\mathrm{CN}$, was not foreseen. This finding possibly relies upon a selection bias, due to the critical conditions of some subjects, who were not testable for cognitive deficits and may suffer from both Dementia and delirium. Further studies are needed to fully assess the relationship between the degree of cognitive impairment, and delirium onset, in hospitalized patients with COVID-19, as well as any possible distinction among different forms of dementia (Alzheimer's disease, frontotemporal dementia, dementia with Lewy bodies, and others).

The possibility to develop delirium due to specific COVID-19 medication (including corticosteroids) has been recently hypothesized [31]. In our sample, the patients treated with corticosteroid therapy had higher severity of delirium. Although this association is commonly reported in other medical conditions [32,33], our study is the first one to assess the association in patients with COVID-19. This finding is relevant, considering the neuroinflammation hypothesis of the "cytokine storm" [2,9], which contributes 
Table 2 Severity of delirium in the different pharmacological subgroups

\begin{tabular}{|c|c|c|c|c|c|c|}
\hline \multirow[t]{2}{*}{ Treatment } & \multicolumn{2}{|c|}{ 4AT score } & \multicolumn{3}{|c|}{ Two-sample independent $t$-test } & \multirow{2}{*}{$\begin{array}{l}\text { Direction } \\
\text { of the } \\
\text { effect }\end{array}$} \\
\hline & Mean & $\mathrm{SD}$ & $t$ value & $\mathrm{df}$ & $p$ & \\
\hline Heparin & & & 1.20 & $1 ; 78$ & 0.23 & / \\
\hline Yes & 9.3 & 2.7 & & & & \\
\hline No & 10 & 2.6 & & & & \\
\hline Hydroxychloroquine & & & 0.45 & $1 ; 78$ & 0.47 & l \\
\hline Yes & 9.8 & 2.5 & & & & \\
\hline No & 9.6 & 2.8 & & & & \\
\hline Antivirals & & & 1.79 & $1 ; 78$ & 0.08 & l \\
\hline Yes & 10.4 & 2.4 & & & & \\
\hline No & 9.3 & 2.8 & & & & \\
\hline Antibiotics & & & 1.81 & $1 ; 78$ & 0.07 & l \\
\hline Yes & 10.1 & 2.3 & & & & \\
\hline No & 8.9 & 3.3 & & & & \\
\hline Corticosteroids & & & 2.60 & $1 ; 78$ & 0.01 & Yes $>$ no \\
\hline Yes & 10.5 & 2.2 & & & & \\
\hline No & 9.0 & 2.8 & & & & \\
\hline Antipsychotics & & & 2.05 & $1 ; 78$ & 0.04 & Yes $>$ no \\
\hline Yes & 10.8 & 2.1 & & & & \\
\hline No & 9.4 & 2.7 & & & & \\
\hline Benzodiazepines & & & 1.43 & $1 ; 78$ & 0.16 & l \\
\hline Yes & 10.4 & 2.0 & & & & \\
\hline No & 9.4 & 2.9 & & & & \\
\hline Antidepressants & & & 0.73 & $1 ; 78$ & 0.47 & / \\
\hline Yes & 10.4 & 2.3 & & & & \\
\hline No & 9.7 & 2.7 & & & & \\
\hline
\end{tabular}

In bold: significant result $(p<0.05)$

\begin{tabular}{lrrrrrrrr}
\hline & & \multicolumn{2}{l}{ 4AT score } & & & \\
\cline { 3 - 4 } & $N$ & Mean & SD & ANOVA & & & Duncan post hoc test \\
\hline Cognitive level & 45 & & & $F$ & df & $p$ & \\
Normal & 25 & 9.6 & 2.7 & & & & \\
Mild cognitive & 12 & 10.8 & 1.3 & & & & \\
$\quad$ impairment (MCI) & & & & & & & \\
Dementia & 8 & 7.6 & 3.7 & & & & \\
\hline
\end{tabular}

Table 3 Cognitive level and severity of delirium by the cross-cutting nature of our evaluation (e.g., in case of unsatisfactory efficacy of other first-line agents, some patients may have received antipsychotics precisely as delirium treatment). Indeed, treatment of delirium in these settings is difficult, considering the possible worsening of COVID-19 symptoms and the risk of drug interaction [37]. Actually, the injectable pharmacological management of delirium includes dexmedetomidine (an alpha 2 agonist), second and third-generation antipsychotics (olanzapine and aripiprazole), and lastly first-generation antipsychotics (haloperidol, promazine, and tiapride). A recent study from dementia [36]. However, this observation could be biased 
Fig. 1 Severity of delirium based on the different cognitive levels at admission. Vertical bars denote the standard error. $(* *)=$ Duncan post hoc test $p<0.01:$ MCI $>$ dementia

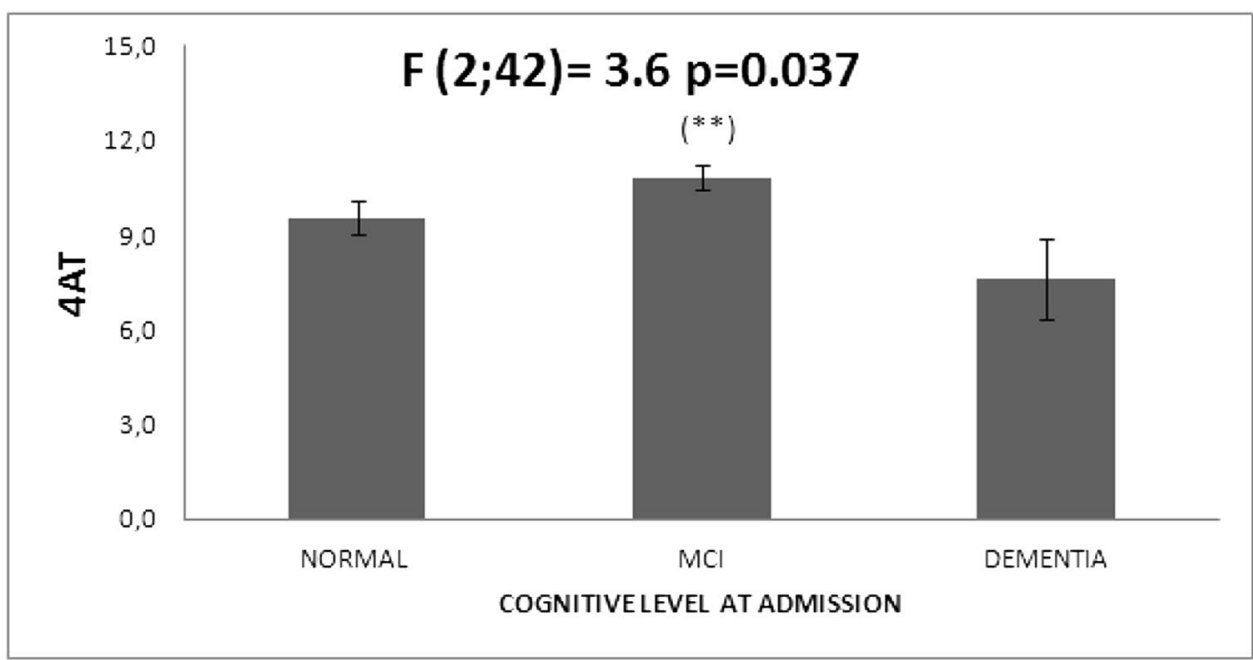

Table 4 Outcome and severity of delirium in the group from Brescia

\begin{tabular}{llllllll}
\hline & & \multicolumn{2}{l}{ 4AT score } & & & \\
\cline { 3 - 4 } & $N$ & Mean & SD & ANOVA & & & Duncan post hoc test \\
\hline Outcome & 38 & & & $F$ & df & $p$ & \\
Survived & 10 & 8.4 & 2.7 & 7.72 & $1 ; 36$ & 0.009 & Deceased $>$ survived \\
Deceased & 28 & 10.7 & 2.1 & & & & \\
\hline
\end{tabular}

ANCOVA with age as covariate: $F(1 ; 35)=3.14 ; p=0.08$ our group has also assessed the greater efficacy and safety of aripiprazole in delirium due to COVID-19 [38]. In addition, risperidone and quetiapine could offer a good choice in the short-term management of delirium in COVID-19 patients [39].

No significant correlations between the administration of antiviral drugs, hydroxychloroquine, benzodiazepines, and the onset of delirium, were found. In this regards our result are in contrast with a recent large study (involving about 2000 patient with delirium/coma in ICU) in which sedation with benzodiazepines was linked to a greater onset of delirium [24].

Low molecular weight heparin is deemed the best anticoagulant choice in COVID-19 patients [40] and was previously reported to decrease the risk for COVID-19-related delirium [23]. However, our results do not confirm this effect. Conversely, polypharmacotherapy (specifically, a greater number of drugs employed) was associated with a higher risk for delirium onset. This new evidence regarding the safety of the singular treatments, particularly neuropsychiatric sequelae, is different from results obtained from other groups [31, 41]. A recent review [42] well summarized the main recommendation of the prolific scientific production of psychiatric management of patients with COVID19. Beside the already discussed mono-pharmacological therapy, also the evaluation of medical framework (kidney and liver function and QTc evaluation), the avoidance of benzodiazepines, the modulation of the therapeutic scheme in progress (if possible), and psychosocial interventions are recommendable choices [42].

Careful therapeutical management to avoid unnecessary complex therapies should therefore be considered.

Regardless of its typology, the presence of delirium is a risk factor for death, consistently with recent observations [43], as confirmed by our research. Therefore, delirium prevention, along with a prompt diagnosis, plays a fundamental role in the prognosis of elderly patients hospitalized for COVID-19 [44]. Delirium onset should call for accurate re-evaluations of the patient's therapy and strict monitoring to avoid possible medical complications. A higher risk of viral transmission, from patients with delirium to the healthcare practitioners, should also be mentioned. In this context, the NICE guidelines on violence and aggression may help clinical management [45]. Delirium assessment should be strongly encouraged in the COVID-19 wards, even earlier than would routinely be considered.

These results of this study should be interpreted with caution given some limitations. The first is the absence of a control group for the analysis on the frequency of delirium onset. Second the results may be influenced by the lack of controls for previous neurologic and psychiatric comorbidities and the correlation with the length of hospitalization. Another limitation of the study is the lack of baseline cognitive 
assessment for many subjects could influence the interpretation of the discussion of MCI influence among 4AT results.

In conclusion, these results provide an insight into the risk/ protective factors in patients hospitalized for COVID-19. Older age, cognitive impairment, the total number of drugs taken, and use of antipsychotics were the most relevant risk factors. Careful prevention of the onset of delirium, with an accurate pharmacological management, along with specific non-pharmacological strategies (e.g., person-centered care or needs driven care, the institution of a "delirium room") [46] could significantly improve the patients' outcomes. New observational studies are needed to confirm these reflections.

Acknowledgements We would thank Elena Butti Lemmi Gigli, Lorenza Cadei, Alessandra Cadeo, Irene Calzavara Pinton, Caterina Cerati, Jacopo Fiori, Giulia Giovannoli, Elisa Piacenza, Arianna Savorelli, Andrea Zucchetti, and Fedele Dono for their precious help during the collecting data phase. The authors wish to dedicate this manuscript in memory of Dr. Sepede. Her energy and scientific keenness will continue to be a reference model for us.

Author contribution Conceptualization: Giovanni Martinotti, Stefano Barlati, Laura Bonanni, Davide Prestia, Claudia Palumbo, Marco Onofrj, Stefano L Sensi, Vita Antonio, Massimo di Giannantonio; methodology: Giovanni Martinotti, Stefano Barlati, Laura Bonanni, Davide Prestia, Claudia Palumbo; formal analysis and investigation: Andrea Miuli, Gianna Sepede, Alice Trabucco, Alessandra Massaro, Martina Olcese, Damiano D'Ardes, Francesco Cipollone, Mario Amore, Emi Bondi, Mirella Russo, Claudia Carrarini; data collection: Andrea Miuli, Alice Trabucco, Alessandra Massaro, Martina Olcese, Damiano D’Ardes, Francesco Cipollone, Mario Amore, Emi Bondi, Mirella Russo, Claudia Carrarini; writing-original draft preparation: Andrea Miuli, Gianna Sepede, Alice Trabucco, Alessandra Massaro, Martina Olcese, Damiano D’Ardes, Francesco Cipollone, Mario Amore, Emi Bondi, Mirella Russo, Claudia Carrarini; writing-review and editing: Giovanni Martinotti, Stefano Barlati, Laura Bonanni, Davide Prestia, Claudia Palumbo; supervision: Marco Onofrj, Stefano L Sensi, Vita Antonio, Massimo di Giannantonio.

\section{Declarations}

Ethical approval This protocol was approved by the Ethics Committee of the Ospedali Civili of Brescia.

Informed consent Informed consent was obtained prior to the interview.

Conflict of interest Giovanni Martinotti has been a consultant and/or a speaker and/or has received research grants from Angelini, Doc Generici, Janssen, Lundbeck, Otsuka, and Pfizer. Stefano Barlati has been a speaker for Angelini, Janssen, Lundbeck, and Otsuka. Mario Amore has been a speaker for GSK. Massimo di Giannantonio has been a consultant and/or a speaker and/or has received research grants from Angelini, Janssen, Lundbeck, Otsuka, Pfizer, and Recordati.

\section{References}

1. Brann DH, Tsukahara T, Weinreb C, Lipovsek M, Van den Berge K, Gong B, et al (2020) Non-neuronal expression of
SARS-CoV-2 entry genes in the olfactory system suggests mechanisms underlying COVID-19-associated anosmia. Sci Adv 6. https://doi.org/10.1126/sciadv.abc5801.

2. Ribeiro DE, Oliveira-Giacomelli Á, Glaser T, Arnaud-Sampaio VF, Andrejew R, Dieckmann L, et al (2020) Hyperactivation of P2X7 receptors as a culprit of COVID-19 neuropathology. Mol Psychiatry:1-16. https://doi.org/10.1038/s41380-020-00965-3.

3. Netland J, Meyerholz DK, Moore S, Cassell M, Perlman S (2008) Severe acute respiratory syndrome coronavirus infection causes neuronal death in the absence of encephalitis in mice transgenic for human ACE2. J Virol 82:7264-7275. https://doi. org/10.1128/JVI.00737-08

4. Ellul MA, Benjamin L, Singh B, Lant S, Michael BD, Easton A et al (2020) Neurological associations of COVID-19. Lancet Neurol 19:767-783. https://doi.org/10.1016/S1474-4422(20) 30221-0

5. Koyuncu OO, Hogue IB, Enquist LW (2013) Virus infections in the nervous system. Cell Host Microbe 13:379-393. https:// doi.org/10.1016/j.chom.2013.03.010

6. Nicholls JM, Butany J, Poon LLM, Chan KH, Beh SL, Poutanen $S$ et al (2006) Time course and cellular localization of SARS-CoV nucleoprotein and RNA in lungs from fatal cases of SARS. PLoS Med 3:e27. https://doi.org/10.1371/journal.pmed.0030027

7. Desforges M, Le Coupanec A, Dubeau P, Bourgouin A, Lajoie L, Dube M, et al (2019) Human coronaviruses and other respiratory viruses: underestimated opportunistic pathogens of the central nervous system? Viruses 12. https://doi.org/10.3390/v12010014.

8. Kempuraj D, Selvakumar GP, Ahmed ME, Raikwar SP, Thangavel R, Khan A et al (2020) COVID-19, mast cells, cytokine storm, psychological stress, and neuroinflammation. Neurosci a Rev J Bringing Neurobiol Neurol Psychiatry 26:402_ 414. https://doi.org/10.1177/1073858420941476

9. Jose RJ, Manuel A (2020) COVID-19 cytokine storm: the interplay between inflammation and coagulation. Lancet Respir Med 8:e46-e47. https://doi.org/10.1016/S2213-2600(20)30216-2

10. Dunkelberger JR, Song W-C (2010) Complement and its role in innate and adaptive immune responses. Cell Res 20:34-50. https://doi.org/10.1038/cr.2009.139

11. Carman KB, Calik M, Karal Y, Isikay S, Kocak O, Ozcelik A et al (2019) Viral etiological causes of febrile seizures for respiratory pathogens (EFES Study). Hum Vaccin Immunother 15:496-502. https://doi.org/10.1080/21645515.2018.1526588

12. Cabeca TK, Bellei N (2012) Human coronavirus NL-63 infection in a Brazilian patient suspected of H1N1 2009 influenza infection: description of a fatal case. J Clin Virol 53:82-84. https://doi.org/10.1016/j.jcv.2011.09.006

13. Nilsson A, Edner N, Albert J, Ternhag A (2020) Fatal encephalitis associated with coronavirus OC43 in an immunocompromised child. Infect Dis (London, England):1-4. https://doi.org/ 10.1080/23744235.2020.1729403.

14. Dessau RB, Lisby G, Frederiksen JL (2001) Coronaviruses in brain tissue from patients with multiple sclerosis. Acta Neuropathol 101:601-604. https://doi.org/10.1007/s004010000331

15. Toniolo S, Di Lorenzo F, Scarioni M, Frederiksen KS, Nobili F (2021) Is the frontal lobe the primary target of SARS-CoV-2? J Alzheimers Dis 81:75-81. https://doi.org/10.3233/JAD-210008

16. Borroni B, Gazzina S, Dono F, Mazzoleni V, Liberini P, Carrarini $C$ et al (2020) Diaphragmatic myoclonus due to SARSCoV-2 infection. Neurol Sci Off J Ital Neurol Soc Ital Soc Clin Neurophysiol 41:3471-3474. https://doi.org/10.1007/ s10072-020-04766-y

17. Dono F, Carrarini C, Russo M, De Angelis MV, Anzellotti F, Onofrj M et al (2021) New-onset refractory status epilepticus (NORSE) in post SARS-CoV-2 autoimmune encephalitis: a case report. Neurol Sci Off J Ital Neurol Soc Ital Soc Clin Neurophysiol 42:35-38. https://doi.org/10.1007/s10072-020-04846-z 
18. Garg RK, Paliwal VK, Gupta A (2021) Encephalopathy in patients with COVID-19: A review. J Med Virol 93:206-222. https://doi. org/10.1002/jmv.26207

19. Helms J, Kremer S, Merdji H, Schenck M, Severac F, Clere-Jehl R et al (2020) Delirium and encephalopathy in severe COVID-19: a cohort analysis of ICU patients. Crit Care 24:491. https://doi.org/ 10.1186/s13054-020-03200-1

20. Qiu H, Tong Z, Ma P, Hu M, Peng Z, Wu W et al (2020) Intensive care during the coronavirus epidemic. Intensive Care Med 46:576-578. https://doi.org/10.1007/s00134-020-05966-y

21. APA. Diagnostic and Statistical Manual, American Psychiatric Association. USA: Arlington; 2013.

22. O'Keeffe ST, Lavan JN (1999) Clinical significance of delirium subtypes in older people. Age Ageing 28:115-119. https://doi.org/ 10.1093/ageing/28.2.115

23. D'Ardes D, Carrarini C, Russo M, Dono F, Speranza R, Digiovanni A, et al (2020) Low molecular weight heparin in COVID-19 patients prevents delirium and shortens hospitalization. Neurol Sci Off J Ital Neurol Soc Ital Soc Clin Neurophysiol:1-4. https://doi. org/10.1007/s10072-020-04887-4.

24. Pun BT, Badenes R, Heras La Calle G, Orun OM, Chen W, Raman $R$ et al (2021) Prevalence and risk factors for delirium in critically ill patients with COVID-19 (COVID-D): a multicentre cohort study. Lancet Respir Med 9:239-50. https://doi.org/10.1016/ S2213-2600(20)30552-X

25. Ely EW, Shintani A, Truman B, Speroff T, Gordon SM, Harrell FEJ et al (2004) Delirium as a predictor of mortality in mechanically ventilated patients in the intensive care unit. JAMA 291:1753-1762. https://doi.org/10.1001/jama.291.14.1753

26. Trautmann S, Rehm J, Wittchen HU (2016) The economic costs of mental disorders: do our societies react appropriately to the burden of mental disorders? EMBO Rep 17:1245-9. https://doi. org/10.15252/embr.201642951

27. Bellelli G, Morandi A, Davis DHJ, Mazzola P, Turco R, Gentile $S$ et al (2014) Validation of the 4AT, a new instrument for rapid delirium screening: a study in 234 hospitalised older people. Age Ageing 43:496-502. https://doi.org/10.1093/ageing/afu021

28. WMA. Dichiarazione di Helsinki della World Medical Association. Evidence 2013;5:1-5.

29. Marcantonio ER (2017) Delirium in hospitalized older adults. $\mathrm{N}$ Engl J Med 377:1456-1466. https://doi.org/10.1056/NEJMcp1605 501

30. Goldberg E, Podell K, Sodickson DK, Fieremans E (2021) The brain after COVID-19: Compensatory neurogenesis or persistent neuroinflammation? EClinicalMedicine 31:100684. https://doi. org/10.1016/j.eclinm.2020.100684

31. Ghasemiyeh P, Borhani-Haghighi A, Karimzadeh I, MohammadiSamani S, Vazin A, Safari A et al (2020) Major neurologic adverse drug reactions, potential drug-drug interactions and pharmacokinetic aspects of drugs used in COVID-19 patients with stroke: a narrative review. Ther Clin Risk Manag 16:595-605. https://doi. org/10.2147/TCRM.S259152

32. Salinas M, Andino P, Palma L, Valencia J, Figueroa E, Ortega J (2021) Early use of corticosteroids in non-critical patients with COVID-19 pneumonia (PREDCOVID): a structured summary of a study protocol for a randomised controlled trial. Trials 22:92. https://doi.org/10.1186/s13063-021-05046-6

33. Saiz-Rodríguez M, Peña T, Lázaro L, González Á, Martínez A, Cordero JA et al (2020) Outpatient treatment of COVID-19 with steroids in the phase of mild pneumonia without the need for admission as an opportunity to modify the course of the disease: a structured summary of a randomised controlled trial. Trials 21:632. https://doi.org/10.1186/s13063-020-04575-w
34. Gupta A, Madhavan MV, Sehgal K, Nair N, Mahajan S, Sehrawat TS et al (2020) Extrapulmonary manifestations of COVID-19. Nat Med 26:1017-1032. https://doi.org/10.1038/s41591-020-0968-3

35. Girard TD, Exline MC, Carson SS, Hough CL, Rock P, Gong MN et al (2018) Haloperidol and ziprasidone for treatment of delirium in critical illness. N Engl J Med 379:2506-2516. https://doi.org/ 10.1056/NEJMoa1808217

36. Morandi A, Zambon A, Di Santo SG, Mazzone A, Cherubini A, Mossello E et al (2020) Understanding factors associated with psychomotor subtypes of delirium in older inpatients with dementia. J Am Med Dir Assoc 21:486-492.e7. https://doi.org/10.1016/j. jamda.2020.02.013

37. Schoen JC, Erlandson KM, Anderson PL (2013) Clinical pharmacokinetics of antiretroviral drugs in older persons. Expert Opin Drug Metab Toxicol 9:573-588. https://doi.org/10.1517/17425 255.2013.781153

38. Martinotti G, Barlati S, Prestia D, Palumbo C, Giordani M, Cuomo A et al (2020) Psychomotor agitation and hyperactive delirium in COVID-19 patients treated with aripiprazole $9.75 \mathrm{mg} / 1.3 \mathrm{ml}$ immediate release. Psychopharmacology (Berl) 237:3497-501. https://doi.org/10.1007/s00213-020-05644-3

39. Ostuzzi G, Gastaldon C, Papola D, Fagiolini A, Dursun S, Taylor D et al (2020) Pharmacological treatment of hyperactive delirium in people with COVID-19: rethinking conventional approaches. Ther Adv Psychopharmacol 10:2045125320942703. https://doi. org/10.1177/2045125320942703

40. Zhou F, Yu T, Du R, Fan G, Liu Y, Liu Z et al (2020) Clinical course and risk factors for mortality of adult inpatients with COVID-19 in Wuhan, China: a retrospective cohort study. Lancet (London, England) 395:1054-1062. https://doi.org/10.1016/ S0140-6736(20)30566-3

41. Wei B, Feng Y, Chen W, Ren D, Xiao D, Chen B (2020) Risk factors for emergence agitation in adults after general anesthesia: a systematic review and meta-analysis. Acta Anaesthesiol Scand. https://doi.org/10.1111/aas.13774

42. Ostuzzi G, Papola D, Gastaldon C, Schoretsanitis G, Bertolini F, Amaddeo F et al (2020) Safety of psychotropic medications in people with COVID-19: evidence review and practical recommendations. Focus (Am Psychiatr Publ) 18:466-481. https://doi. org/10.1176/appi.focus. 18308

43. Marengoni A, Zucchelli A, Grande G, Fratiglioni L, Rizzuto D (2020) The impact of delirium on outcomes for older adults hospitalised with COVID-19. Age Ageing 49:923-926. https://doi. org/10.1093/ageing/afaa189

44. LaHue SC, James TC, Newman JC, Esmaili AM, Ormseth CH, Ely EW (2020) Collaborative delirium prevention in the age of COVID-19. J Am Geriatr Soc 68:947-949. https://doi.org/10. 1111 /jgs. 16480

45. National Institute for Health and Care Excellence. Violence and aggression: short-term management in mental health, health and community settings 2015. https://www.nice.org.uk/guidance/ng10 (Accessed 25 Jan 2021).

46. Keszycki RM, Fisher DW, Dong H (2019) The HyperactivityImpulsivity-Irritiability-disinhibition-aggression-agitation domain in Alzheimer's disease: current management and future directions. Front Pharmacol 10:1109. https://doi.org/10.3389/fphar.2019. 01109

Publisher's note Springer Nature remains neutral with regard to jurisdictional claims in published maps and institutional affiliations. 